\title{
Trois poèmes des Fleurs du mal en espagnol : un exercice de traduction comparée
}

\section{Elisa Martín Ortega}

\section{(2) OpenEdition}

Journals

Édition électronique

URL : http://journals.openedition.org/rief/613

DOI : $10.4000 /$ rief.613

ISSN : 2240-7456

Éditeur

Seminario di filologia francese

Référence électronique

Elisa Martín Ortega, "Trois poèmes des Fleurs du mal en espagnol : un exercice de traduction comparée », Revue italienne d'études françaises [En ligne], 4 | 2014, mis en ligne le 15 décembre 2014, consulté le 19 avril 2019. URL : http://journals.openedition.org/rief/613 ; DOI : 10.4000/rief.613

Ce document a été généré automatiquement le 19 avril 2019

\section{(c) (i) (9)}

Les contenus de la RIEF sont mis à disposition selon les termes de la Licence Creative Commons Attribution - Pas d'Utilisation Commerciale - Pas de Modification 4.0 International. 


\title{
Trois poèmes des Fleurs du mal en espagnol : un exercice de traduction comparée
}

\author{
Elisa Martín Ortega
}

\section{Introduction}

1 Toute traduction est une forme d'interprétation. Le traducteur doit se servir des ressources que lui offre sa langue maternelle, qui seront dans certains cas différentes de celles de la langue originale du texte. Dans le cas de l'espagnol, par rapport au français, il faudrait surtout souligner l'hyperbate, qui est permis avec plus de naturel et de fréquence qu'en français, et le rythme accentuel, qui provient de la combinaison d'oxytons ("agudas"), de paroxytons ("llanas") et de proparoxytons ("esdrújulas").

2 Le respect du sens original des poèmes n'implique pas forcément le fait d'en faire une copie. En ce qui concerne la métrique, les trois poèmes que j'ai traduits (Correspondances, L'ennemi et Tristesses de la lune) sont des sonnets écrits en alexandrins. Le symbolisme représente un moment de profonde rénovation de la métrique française : quarante-et-un des soixante-seize sonnets de Les fleurs du mal sont appelés «sonnets libertins", car la distribution des rimes n'y suit pas le schéma traditionnel. L'équivalent espagnol de ce mouvement est le « Modernisme », incarné dans la figure de Rubén Darío, qui entreprit la plus grande rénovation de la poésie espagnole depuis la Renaissance.

3 En espagnol, les sonnets sont traditionnellement composés de vers hendécasyllabes, et le schéma de la rime est assez régulier (ABBA ABBA CDC DED, ou bien CDE DED, la rime des tercets étant la plus libre). Rubén Darío, toutefois, a commencé à composer des sonnets en alexandrins espagnols (vers de quatorze syllabes avec une césure à la septième). C'est pourquoi j'ai décidé de traduire les sonnets de Baudelaire en vers alexandrins : cela fait penser au mouvement « moderniste » et en même temps cela permet plus de naturel dans la traduction, car souvent le français est une langue plus compacte que l'espagnol. 
4 La question du maintien des rimes pose un grand dilemme au traducteur. Les supprimer implique, sans doute, un renoncement, surtout s'il s'agit d'un poète comme Baudelaire qui, fondateur du symbolisme, accorde l'importance que l'on sait à la musicalité des vers. Néanmoins, beaucoup de traducteurs ont opté pour le vers blanc, étant donné que dans beaucoup de cas le maintien de la rime pousse à des constructions grammaticales complexes et forcées, ou encore à trahir le sens original. En même temps, il ne faut pas oublier que Baudelaire lui-même affirmait que la rime, loin d'être une limitation pour le poète, donne des ailes à l'imagination. Le traducteur, bien sûr, n'est pas censé inventer, mais la suppression de la rime n'est-elle pas une forme de trahison?

Avant tout, il faut examiner les particularités des rimes espagnole et française. En espagnol on distingue deux types de rimes: la rime par assonance, ou rime pauvre suivant la terminologie française (seules les voyelles coïncident à partir de la dernière syllabe accentuée; elle est caractéristique de la poésie populaire), et la rime "consonante", ou rime riche (les voyelles et les consonnes coïncident à partir de la dernière syllabe accentuée; elle est caractéristique de la poésie cultivée). En français, cette différenciation est moins nette, mais, comme on le sait, on distingue rimes masculines et féminines, généralement alternées.

6 Étant donné que je ne voulais pas renoncer à la rime, et qu'une traduction avec rimes riches (consonantes) provoquerait des infidélités au texte et forcerait la syntaxe, j'ai décidé de maintenir la distribution originale des rimes mais en utilisant des rimes par assonances (pauvres). Cela est sans doute discutable, car le sonnet traditionnel en espagnol se compose toujours avec la rime "consonante". Même Ruben Darío, le grand rénovateur, respectait cette règle. Néanmoins, il m’a semblé que la rime par assonances donne au traducteur une liberté suffisante pour ne pas trahir le sens, et permet en même temps de sauvegarder la musicalité du poème.

7 Je présenterai, à côté de la version originale française, la traduction que je propose, suivie d'autres traductions en espagnol du même poème. J'offre ensuite un commentaire de chaque poème, en expliquant les difficultés de sa traduction et les solutions adoptées par les différents traducteurs.

8 La plupart des traducteurs utilisent aussi le vers alexandrin espagnol (14 syllabes). Deux d'entre eux, Luis Martínez de Merlo et Antonio Martínez Sarrión, emploient le vers blanc (sans rime mais avec un schéma métrique), même si ils créent parfois des assonances pour renforcer le rythme.

9 Nydia Lamarque traduit avec rime "consonante". Parfois, elle s'éloigne de l'original ou crée des expressions un peu forcées pour conserver la rime. Arturo Fruttero propose la même solution.

10 Luis Antonio de Villena, pour sa part, présente une traduction avec des vers métriquement différents (ils n'ont pas tous le même nombre de syllabes), et sans rime. Il explique dans l'introduction à sa traduction qu'il voudrait émuler le vers libre que les symbolistes ont introduit dans la poésie française.

11 Je n'ai donc pas essayé de faire une compilation de toutes les traductions des Fleurs du mal en espagnol, ce qui aurait été l'objet d'un autre travail. J'en ai simplement réuni quelquesunes qui puissent illustrer les différentes options et les critères de la traduction. Dans ma traduction j'ai suivi, à tout moment, les critères présentés dans cette introduction. 


\section{Correspondances / Correspondencias}

La Nature est un temple où de vivants piliers

Laissent parfois sortir de confuses paroles ;

L'homme y passe à travers des forêts de symboles

Qui l'observent avec des regards familiers.

Comme de longs échos qui de loin se confondent

Dans une ténébreuse et profonde unité,

Vaste comme la nuit et comme la clarté,

Les parfums, les couleurs et les sons se répondent.

Il est des parfums frais comme des chairs d'enfants,

Doux comme les hautbois, verts comme les prairies,

- Et d'autres, corrompus, riches et triomphants,

Ayant l'expansion des choses infinies,

Comme l'ambre, le musc, le benjoin et l'encens,

Qui chantent les transports de l'esprit et des sens.

Traduction de Elisa Martín Ortega

Naturaleza es un templo en que vivos pilares

Dejan salir a veces sus palabras confusas ;

Entre bosques de símbolos el ser humano cruza,

Mientras éstos le observan con ojos familiares.

Como ecos prolongados que a lo lejos se mezclan

En una tenebrosa y profunda unidad,

Vasta como la noche y como la claridad,

Perfumes y colores, y sones se contestan.

Hay perfumes $\tan$ frescos como carnes de niños,

Tan dulces como oboes, como los prados verdes,

-Y hay otros corrompidos, y triunfantes y ricos,

Que igual que el infinito, sin límite se extienden.

Como el benjuí, el incienso, el almizcle y el ámbar

Cantan el acuerdo de los sentidos y el alma.

Traduction de Luis Martínez de Merlo

La Creación es un templo de pilares vivientes

que a veces salir dejan sus palabras confusas ;

el hombre la atraviesa entre bosques de símbolos

que le contemplan con miradas familiares.

Como los largos ecos que de lejos se mezclan

en una tenebrosa y profunda unidad,

vasta como la luz, como la noche vasta,

se responden sonidos, colores y perfumes.

Hay perfumes tan frescos como carnes de niños, dulces tal los oboes, verdes tal las praderas

- y hay otros, corrompidos, ricos y triunfantes, que tienen la expansión de las cosas infinitas, como el almizcle, el ámbar, el benjuí y el incienso, que cantan los transportes de sentidos y espíritu.

Traduction de Nydia Lamarque 
Naturaleza es templo donde vivos pilares

Dejan salir a veces tal cual palabra oscura ;

Entre bosques de símbolos va el hombre a la ventura,

Que lo contemplan con miradas familiares.

Como ecos prolongados, desde lejos fundidos

En una tenebrosa y profunda unidad,

Vasta como la noche y cual la claridad,

Se responden perfumes, colores y sonidos.

Así hay perfumes frescos como carnes de infantes,

Verdes como praderas, dulces como el oboe.

-Y hay otros corrompidos, y ricos y triunfantes,

De una expansión de cosas infinitas henchidos,

Como el almizcle, el ámbar, el incienso, el aloe,

Que cantan los transportes del alma y los sentidos.

Traduction de Arturo Fruttero

El Mundo es como un templo donde vivos pilares

Dejan salir a veces palabras reticentes ;

Cruza el hombre por selvas de símbolos videntes

Que le observan con hondas miradas familiares.

Como los largos ecos que a lo lejos se atestan

En una tenebrosa y profunda unidad,

Inmensa cual la noche y cual la claridad,

Perfumes y colores y sones se contestan.

Hay perfumes $\tan$ frescos como carnes niñas,

Suaves como los oboes, verdes como campiñas ;

$Y$ hay fragancias corruptas, triunfantes y exquisitas,

Con la expansión que tienen las cosas infinitas,

Como el benjuí, el almizle, el ámbar y el incienso,

Que cantan los arrobos del sentido en suspenso.

Traduction de Luis Antonio de Villena

La Natura es templo en que pilares vivos

dejan salir, a veces, confusas palabras ;

pasa en él el hombre por bosques de símbolos

que le observan con miradas familiares.

Como largos ecos que a lo lejos se mezclan

en una tenebrosa y profunda unidad,

vasta como la noche y cual la claridad,

perfumes, colores y son se responden.

Hay perfumes frescos como carne de niño.

Dulces como oboes, verdes como praderas.

$\mathrm{Y}$ otros corrompidos, triunfales y ricos,

con la expansión propia de las cosas infinitas,

como el ámbar, benjuí, almizcle o el incienso,

que cantan los transportes del alma y los sentidos.

12 Commentaire

13 Correspondances est un des poèmes les plus emblématiques du symbolisme. Il est construit sur le concept d'analogie universelle : la nature possède une âme, parle avec un langage 
caché, et l'homme essaye d'entretenir avec elle, à travers son imagination, une relation de réciprocité, de vraie communication :

L'imagination ne se trouve pas dans l'homme, mais elle est l'esprit du lieu et du moment ; ce n'est pas seulement la force à travers laquelle on voit la réalité visible et la réalité cachée ; elle est aussi le moyen par lequel la nature, à travers le regard $\mathrm{du}$ poète, se regarde. Par l'imagination la nature nous parle et parle avec ellemême.

[La imaginación no está en el hombre, sino que es espíritu del lugar y del momento ; no es sólo la potencia por la que vemos la realidad visible y la oculta; también es el medio por el que la naturaleza, a través de la mirada del poeta, se mira. Por la imaginación la naturaleza nos habla y habla con ella misma.] $]^{1}$

La création est considérée comme un dialogue qui permet de réinventer la réalité, et l'imagination n'est pas une qualité humaine, mais elle a une existence autonome. L'artiste serait celui qui est capable de regarder la nature avec ses propres yeux et d'y trouver la partie visible et celle qui est cachée, pour en faire une métaphore.

L'analogie universelle repose sur l'idée qu'il n'y a pas de faits ou de phénomènes isolés. Les couleurs, les parfums et les sons vivent dans une mystérieuse correspondance, qui en littérature est exprimée à travers la synesthésie, figure rhétorique préférée des symbolistes. Le poème Correspondances peut ainsi être compris comme une énorme synesthésie, dans laquelle le sens qui finalement prédomine est l'odorat (le chant aux parfums est constant dans la poésie de Baudelaire). Les odeurs se répandent sans limite, elles envahissent le milieu et provoquent l'union du corps et de l'âme, des sens et des sentiments, qui font également partie de cette grande analogie.

Pour traduire ce poème, il faut considérer l'importance du rythme et des rimes dans l'affirmation de son contenu. Bien sûr, il s'agit d'une caractéristique de tous les poèmes de Baudelaire, et en général des symbolistes, qui font recours à des synesthésies et à la musique du langage pour exemplifier des telles correspondances. Cependant, dans ce cas particulier, une traduction qui sacrifie l'harmonie formelle en faveur du contenu aurait manqué complètement son but. On pourrait même oser dire que le maintien de la rime est essentiel : le poème évoque des regards qui se trouvent, des échos qui se mélangent, un bal des sens qui se répondent. Et la rime est un recours très efficace pour exprimer ces clins d'œil et ces répétitions, semblables mais jamais identiques.

Du point de vue de la métrique, la structure du sonnet répond au schéma suivant : AbbA cDDc EfE fGG. Les deux quatrains alternent des rimes masculines et féminines différentes, et les tercets introduisent deux nouvelles rimes croisées pour finir avec un sentencieux distique de rimes plates. Le schéma rythmique évoque le sonnet anglais, fait de trois quatrains et d'un distique de rimes plates. Ce genre de clôture est assez habituel dans les sonnets de Baudelaire.

Dans ma traduction j'ai maintenu le schéma, avec des rimes par assonance et paroxytones, à l'exception des deux vers intérieurs du second quatrain qui ont une rime en - $a$ final accentué. Cependant, Nydia Lamarque choisit de remplacer la rime des tercets par le schéma le plus typique des sonnets espagnols: DED EFE, mais ce faisant elle renonce à maintenir à la fin le distique de rimes plates, qui donne une plus grande force au poème. Arturo Fruttero opte pour le schéma : DDE EFF, trois distiques de rimes plates de suite, qui génèrent une sonorité un peu répétitive, mais qui permettent une plus grande fidélité au texte original, comme on le verra plus tard. 
19 Le début du poème pose un premier problème au traducteur: «La Nature ». "Naturaleza », en espagnol, est un mot beaucoup plus long que son équivalent français, ce qui provoque des problèmes de métrique. Le fait qu'il apparaisse avec majuscule dans l'original suggère une personnification de cette force motrice : la nature conçue comme déesse, comme principe actif générateur de la vie. En plus, tout au long du poème, on assiste à l'affirmation de l'idée d'une nature vivante, qui ressent et qui communique avec l'homme. Elle n'est pas d'un objet, mais un sujet qui agit au même niveau que l'être humain. C'est pour cela que j'ai décidé de retirer l'article et de commencer le poème avec le mot « Naturaleza ». D'autres traducteurs ont préféré remplacer « La Naturaleza » par «La Creación » et «El Mundo ». J'ai considéré que le terme naturaleza était plus approprié pour nommer l'espace où l'analogie universelle a lieu, étant donné que deux vers plus tard le poète parle de "forêts de symboles». "Creación» a quelques connotations religieuses que je ne vois pas dans le poème original, et « el Mundo » peut difficilement être considéré un sujet qui interagisse avec l'homme. Luis Antonio de Villena propose le remplacement du terme par «La Natura », un latinisme qui pourrait être accepté dans ce cas précis (la déesse nature), mais qui contraste toujours avec le choix plus simple du terme français « Nature ».

Cela dit, les deux quatrains admettent une traduction en alexandrins espagnols (vers de 14 syllabes) avec rime riche sans trop de complexité : de petites hyperbates (« perfumes y colores, y sones se contestan»), et quelque remplacement par des synonymes (" el ser humano » par « el hombre »), permettent de faire cadrer le tout.

21 Néanmoins, le début des tercets pose un problème aux traductions en rime riche : « Il est des parfums frais comme des chairs d'enfants». Je traduis stricto sensu en ajoutant l'adverbe «tan » pour compléter le premier hémistiche : « Hay perfumes tan frescos como carnes de niños » (ce vers coïncide exactement avec la traduction de Luis Martínez de Merlo). C'est, sans doute, l'option la plus fidèle à l'original et la plus naturelle en espagnol. Cependant, la difficulté de trouver des rimes riches avec -iño ou -iños porte Nydia Lamarque à remplacer « niños » par « infantes », parole qui rime facilement avec «triunfantes », comme dans l'original.

Il est vrai que le mot "infantes » a la même racine étymologique que le français " enfants ", mais son usage dans chacune des deux langues est très différent. " Infante ", en espagnol, est un terme désuet ou vieilli pour faire référence à un enfant, et il fait penser au vocabulaire de la royauté et des titres nobiliaires. Cependant, il faut remarquer la solution adoptée par Arturo Fruttero. Il change la distribution originale des rimes et fait rimer les deux premiers vers du premier tercet : « carnes niñas » avec « campiñas ». Il s'agit d'une proposition intéressante, même si l'on a déjà mentionné le discutable effet sonore que provoque la succession de trois distiques de rimes plates dans les tercets. En effet, dans les sonnets français classiques presque toutes les combinaisons des rimes étaient acceptées dans les tercets, l'une d'entre elles au moins étant croisée. Dans ma traduction, j'ai pu maintenir « carnes de niños » car, en utilisant la rime par assonance, j'ai dû modifier uniquement l'ordre des termes des deux vers suivants, et laisser à la fin le mot « ricos ». Luis Antonio de Villena trouve une solution similaire : il ne s'impose aucun schéma métrique préétabli, mais recourt souvent à des assonances pour renforcer le rythme du poème.

23 Le premier vers du second tercet est, probablement, celui pour lequel je me suis permis une traduction plus libre. Presque tous les traducteurs optent pour un haut degré de littéralité dans ce cas : " que tienen la expansión de cosas infinitas ", écrit Luis Martínez 
de Merlo; et les autres versions sont très semblables, si l'on excepte, peut-être, celle de Nydia Lamarque, qui traduit: "de una expansión de cosas infinitas henchidos». Elle ajoute un participe à la fin, qui lui est utile pour la rime, et remplace le «ayant » avec lequel commence l'original. L'idée est identique, même si la formulation est probablement moins claire. Je me suis trouvée, ici, face à un dilemme: une version littérale ne me permettait en aucun cas de maintenir la distribution des rimes de l'original (je n'ai pas trouvé moyen de faire rimer, même par assonance, « infinitas » avec " praderas », « verdes » ou " oboes »). En même temps, il s'agit d'un vers hermétique, qui donne lieu à une expression qui n'est pas naturelle en espagnol. J'ai donc décidé de reformuler l'idée, et de remplacer "se expanden" par "se extienden", ce qui me permettait la rime avec «verdes». Je crois que je n'ai pas trahi le sens que Baudelaire a exprimé dans ce vers; ce faisant, j'ai cherché, en tout cas, une option qui semblait naturelle pour le lecteur hispanophone.

Dans le distique final de rimes plates les traductions varient la distribution des éléments nommés : ambre, musc, benjoin et encens, et quelques-unes d'entre elles remplacent " espíritu » par " alma », d'après les nécessités métriques ou de la rime - étant donné que dans ce cas le deux mots peuvent être considérés synonymes. Arturo Fruttero offre ici une version très éloignée du texte français: "que cantan los arrobos del sentido en suspenso ». Je ne crois pas que l'idée de l'original soit bien conservée, et le terme " alma » manque comme opposition à "sentidos", ce qui est essentiel pour comprendre le contenu du poème. Néanmoins, dans ma traduction ce dernier vers apparaît également un peu transformé, interprété. Au lieu de « que cantan los transportes de los sentidos y el espíritu », je propose " cantan el acuerdo de los sentidos y el alma ». Il m'a semblé que le pronom relatif "que» était accessoire, étant donné qu'il fait fonction de sujet et remplace l'énumération immédiatement antérieure. D’un autre côté, la notion de «transporte» en espagnol fait plus penser à un déplacement physique et spatial que le français "transports", appliqué fréquemment à des changements psychiques ou animiques. Ainsi, j'ai décidé de le remplacer par « acuerdo », interprétant que l'échange et l'analogie universelle donnent lieu à une confusion et, encore plus, à une union, du physique et de l'animique, de la perception et des sentiments.

\section{L'ennemi / El enemigo}

Ma jeunesse ne fut qu'un ténébreux orage,

Traversé çà et là par de brillants soleils ;

Le tonnerre et la pluie ont fait un tel ravage,

Qu'il reste en mon jardin bien peu de fruits vermeils.

Voilà que j'ai touché l'automne des idées,

Et qu'il faut employer la pelle et les râteaux

Pour rassembler à neuf les terres inondées,

Où l'eau creuse des trous grands comme des tombeaux.

Et qui sait si les fleurs nouvelles que je rêve

Trouveront dans ce sol lavé comme une grève

Le mystique aliment qui ferait leur vigueur?

- Ô douleur ! ô douleur ! Le Temps mange la vie,

Et l'obscur Ennemi qui nous ronge le cœur

Du sang que nous perdons croît et se fortifie! 


\section{Traduction de Elisa Martín Ortega}

Mi juventud fue sólo una tormenta oscura, A veces traspasada por el brillo del sol ; Han hecho tal estrago los truenos y la lluvia Que en mi jardín escasos frutos hay ya en sazón. De las ideas hoy he alcanzado el otoño, $\mathrm{Y}$ es necesario usar el rastrillo y la pala Para allanar de nuevo los campos anegados, En donde cava hoyos como tumbas el agua. ¿Quién sabe si las nuevas flores con las que sueño Hallarán en mi suelo, como una playa yermo, El alimento místico que les dará vigor? -¡Oh dolor ! ¡oh dolor ! ¡Come el Tiempo la vida, Y el oscuro Enemigo que roe el corazón Crece y se fortifica con la sangre perdida! Traduction de Luis Martínez de Merlo Mi juventud tan sólo fue una negra tormenta, cruzada aquí y allá por soles luminosos ; tal estrago en mí han hecho los rayos y la lluvia, que en mi jardín ya quedan muy pocos frutos rojos. Y heme que ya el otoño toqué de las ideas, y es menester usar la pala y los rastrillos para igualar de nuevo las tierras inundadas, donde el agua ha cavado grandes hoyos cual tumbas. ¿Encontrarán las nuevas flores con las que sueño, en este suelo igual que una playa empapado el alimento místico que ha de darles vigor? -¡Oh dolor! ¡Oh dolor ! ¡Come el Tiempo a la vida, y el oscuro enemigo que el corazón nos roe se fortifica y crece robándonos la sangre!

\section{Traduction de Nydia Lamarque}

Mi juventud fue sólo tenebrosa tormenta, De fulgurantes soles cruzada aquí y allá ; Fue de rayos y lluvias la obra tan violenta, Que en mi jardín hay pocos frutos bermejos ya. Y hoy al otoño de las ideas he llegado, $Y$ ahora debo el rastrillo y la pala esgrimir, Para alisar de nuevo el terreno inundado, Donde el agua agujeros como tumbas fue a abrir. ¿Y quién sabe si, a esas flores nuevas que ensaya Mi sueño, da este suelo, yermo como una playa, El místico alimento que haría su esplendor? -Come el Tiempo la vida, joh dolor ! joh dolor ! ¡Y el oscuro Enemigo que el corazón nos roe, Con nuestra propia sangre crece y cobra vigor ! Traduction de Antonio Martínez Sarrión Mi juventud no fue sino un gran temporal Atravesado, a rachas, por soles cegadores ; 
Hicieron tal destrozo los vientos y aguaceros

Que apenas, en mi huerto, queda un fruto en sazón.

He alcanzado el otoño total del pensamiento,

$\mathrm{Y}$ es necesario ahora usar pala y rastrillo

Para poner a flote las anegadas tierras

Donde se abrieron huecos, inmensos como tumbas.

¿Quién sabe si los nuevos brotes en los que sueño

Hallarán en mi suelo, yermo como una playa,

El místico alimento que les dará vigor?

¡ Oh dolor ! ¡Oh dolor! Devora vida el Tiempo,

Y el oscuro Enemigo que nos roe el corazón,

Crece y se fortifica con nuestra propia sangre. 
avec le monde rural. Baudelaire parle de «rassembler à neuf les terres inondées ». Dans ma traduction: "allanar de nuevo los campos inundados ». Luis Martínez de Merlo et Nydia Lamarque écrivent «alisar ». L'idée est celle de redonner de la consistance a des terres abîmées par les pluies (l'orage juvénile), transformées en boue, parsemées de trous. Seul Antonio Martínez Sarrión s'éloigne de cette interprétation : «Para poner a flote las anegadas tierras ». Cependant, "mettre à flottage» les terres est une image un peu étrange, car la terre ne peut flotter, même si l'image permet d'interpréter l'idée de séparation des terres et des eaux.

31 Dans le deuxième vers du premier tercet j'ai créé une rime interne qui n'apparait pas dans l'original. Il m'a semblé qu'elle était utile pour renforcer le rythme, étant donné que les deux premiers vers du premier tercet riment entre eux («sueño», «suelo», «yermo »). Dans l'original il y a une claire rime interne entre le premier et le second tercet - «vigueur » et « douleur », répété deux fois - que presque toutes les traductions maintiennent intacte (car elle peut être reportée en espagnol sans faire aucun changement).

"Le temps mange la vie», écrit ensuite Baudelaire. Il est certain qu'en espagnol la traduction la plus convenable serait «El tiempo devora la vida », solution à laquelle se rapproche Antonio Martínez Sarrión en éliminant l'article. À cause des exigences de la métrique, j'ai opté pour la littéralité dans ce cas : « Come el Tiempo la vida ».

Dans le dernier vers, j'ai considéré que le « sang perdu » est, évidemment, le sang que l'on perd, auquel fait référence Baudelaire. J'ai créé, dans ce vers, une autre rime interne: "Crece y se fortifica con la sangre perdida », qui m’a semblé adéquate pour souligner la fin sentencieuse, étant donné que dans ce poème les deux derniers vers n'ont pas de rimes plates.

\section{Tristesses de la lune / Tristezas de la luna}

Ce soir, la lune rêve avec plus de paresse ;

Ainsi qu'une beauté, sur de nombreux coussins, Qui d'une main distraite et légère caresse

Avant de s'endormir le contour de ses seins, Sur le dos satiné des molles avalanches, Mourante, elle se livre aux longues pâmoisons, Et promène ses yeux sur les visions blanches Qui montent dans l'azur comme des floraisons. Quand parfois sur ce globe, en sa langueur oisive, Elle laisse filer une larme furtive, Un poète pieux, ennemi du sommeil, Dans le creux de sa main prend cette larme pâle, Aux reflets irisés comme un fragment d'opale, Et la met dans son cœur loin des yeux du soleil. Traduction de Elisa Martín Ortega Esta noche la luna sueña con más pereza ; Cual una mujer bella, sobre varios cojines, Que acaricia con mano distraída y ligera La línea de sus senos cuando empieza a dormirse, 
Sobre el tacto ondulado de la sedosa espalda, Agónica se entrega a largos paroxismos, Y pasea sus ojos por las visiones blancas Que ascienden al azul cual ramos florecidos. Cuando, sobre la tierra, deja, ociosa y lánguida, Ciertas veces caer una furtiva lágrima Un poeta piadoso, enemigo del sueño, En su mano recoge esta lágrima pálida, Cual fragmento de ópalo, de irisados reflejos, Y ocultándola al sol, la introduce en su pecho. Traduction de Luis Martínez de Merlo Esta noche, la luna sueña más perezosa ; lo mismo que una bella sobre muchos cojines, que acaricia con mano ligera y distraída, antes de adormecerse, la línea de sus senos, sobre el brillante dorso de blandas avalanchas, en un largo desmayo, muriente se abandona, y pasea sus ojos por las blancas visiones que como floraciones en el azul se alzan. Cuando sobre este globo, en sus lánguidos ocios deja caer, a veces, furtiva alguna lágrima, un poeta devoto, y enemigo del sueño, en su mano recoge esta lágrima pálida, de irisados reflejos, tal fragmentos de un ópalo, y a los ojos del sol en su pecho la oculta.

\section{Traduction de Nydia Lamarque}

Esta noche la Luna sueña con más pereza ; Como en cojines reclinada una belleza Que acariciando leve, con mano distraída De su seno el contorno, se va a quedar dormida. Sobre el dorso de seda de los suaves nublados, Moribunda, se entrega a éxtasis prolongados, Y pasea sus ojos por las blancas visiones Que en el azul ascienden tal como floraciones. Cuando sobre este mundo su languidez ociosa Deja caer alguna lágrima sigilosa, Un poeta piadoso, de dormir enemigo, De su mano en el hueco esta lágrima fría Toma, fragmento de ópalo que al iris desafía, Y en su corazón, lejos del sol, le presta abrigo. Traduction de Antonio Martínez Sarrión Esta noche la luna sueña con más pereza, Cual si fuera una bella hundida entre cojines Que acaricia con mano discreta y ligerísima, Antes de adormecerse, el contorno del seno. Sobre el dorso de seda de aludes deslizantes, Moribunda, se entrega a prolongados éxtasis, 
Y pasea su mirada sobre visiones blancas,

Que ascienden al azul igual que floraciones.

Cuando sobre este globo, con languidez ociosa,

Ella deja rodar una furtiva lágrima,

Un piadoso poeta, enemigo del sueño,

De su mano en el hueco, coge la fría gota

Como un fragmento de ópalo de irisados reflejos.

Y la guarda en su pecho, lejos del sol voraz.

\section{Commentaire}

La lune est la compagne du poète. Elle lui révèle, dans les nuits d'insomnie, ses multiples secrets. En plus, elle est femme, et comme telle elle possède une puissante charge sensuelle, qui apparaît clairement reflétée dans ce poème. La lune et la femme se fondent et se séparent tout au long des vers. Elles sont mélancoliques, pleines d'érotisme, et le poète devient leur complice. Il est celui qui connaît et recueille leurs mystères (enfermés dans une larme qui tombe), pour les occulter à la vue du monde diurne. Le poète et la lune sont des créatures nocturnes, plongés en eux-mêmes, tristes et désireux.

Le schéma métrique est très semblable à celui des sonnets antérieurs, avec une alternance parfaite de rimes masculines et féminines: $\mathrm{aBaB} \mathrm{cDcD}$ eeF ggF. Pour le traduire, j'ai modifié la distribution des rimes dans les tercets : EEF EFF. J'ai pris cette décision pour ne pas forcer l'expression sans être infidèle à la lettre et, en même temps, parce que j'ai voulu maintenir une haute concentration de mots accentués sur l'antépénultième syllabe dans ces tercets, incitée par la répétition de la parole «lágrima », et l'apparition de « pálida », « ópalo » et « lánguida ». La rime représentée par la lettre E est formée par ces trois mots accentués sur l'antépénultième syllabe (« lánguida », « lágrima » et " pálida »), créant un rythme avec lequel j'ai voulu rappeler la prédilection que les écrivains modernistes ont eue pour ce genre de mots.

La lune rêve avec paresse. Elle est comparée, à ce moment-là, avec " une beauté »: littéralement «belleza », mais qui ici fait clairement référence à une belle femme (plus tard sont mentionnés ses seins). Dans le poème La beauté des Fleurs du mal, la beauté apparaît incarnée en une déesse parfaite qui rend les poètes amoureux avec ses yeux. Néanmoins, au contraire de la lune, elle est pure luminosité, faite de marbre, et jamais elle ne permettrait qu'une larme se glisse sur ses joues :

Je trône dans l'azur comme un sphinx incompris,

J'unis un cœur de neige à la blancheur des cygnes,

Je hais le mouvement qui déplace les lignes,

Et jamais je ne pleure et jamais je ne ris. contour de ses seins. Pour les exigences de la métrique, j'ai remplacé « el contorno » par « la ligne de ses seins », comme le fait également Luis Martínez de Merlo.

Le premier vers du second quatrain présente des difficultés d'interprétation et de traduction. L'option la plus littérale est « sobre el brillante dorso de blandas avalanchas » (Luis Martínez de Merlo), qui cependant me semble compliquée à comprendre en espagnol. Le mot français « dos » fait référence au « dorso » et à la « espalda » humaine, et Baudelaire parle d'une femme qui est en même temps la lune. C'est là le problème. Dans ce cas, j'ai opté pour traduire « espalda ", et mettre en relation l'image avec la figure de la femme, car dans le vers antérieur on trouve une allusion aux seins, et le vers suivant parle de l'extase et des évanouissements. "Sobre el tacto ondulado de la sedosa 
espalda » : les avalanches molles ou glissantes font ici référence aux courbes du dos doux sur lesquelles elle est couchée.

La jeune femme, "mourante", s'abandonne à l'extase, aux paroxysmes et aux évanouissements. L'adjectif «moribunda », cependant, m'a semblé excessif dans ce cas. En espagnol il a une connotation obscure et même morbide qui n'est pas partagée par le mot français «mourante ». C'est pourquoi j'ai décidé de le remplacer par « agónica », un mot synonyme, mais qui admet plus facilement un sens figuré.

La lune observe les nuages, "blancas visiones", qui "ascienden al azul igual que floraciones» (Antonio Martínez Sarrión). La floraison est un acte, un moment qui se repète graduellement dans la vie des arbres et des plantes. Un synonyme en espagnol serait «florecimiento». Et comment pourrait monter un «florecimiento"? Trouvant cette image très étrange en espagnol, j'ai substitué «floraciones» par «ramos florecidos ».

Dans le premier tercet la lune laisse tomber une larme sur le globe terrestre. Le poème originel dit "ce globe", option maintenue par deux des traductions. Nydia Lamarque écrit " este mundo », et j'ai décidé de traduire « la tierra », car c'est le nom de la planète. En plus, «tierra » évoque plus l'idée de la sphère : normalement on parle en espagnol de la rotondité de la terre, et non du monde rond.

Ceci dit, j'ai déjà expliqué au début que, dans les tercets, j'ai essayé de créer un rythme basé sur l'accumulation des proparoxytons. La fin du poème est un distique de rimes plates (ce qui n'est pas le cas dans l'original), et il y a une certaine économie des mots que j'ai considérés non indispensables pour faire concorder la métrique. En ce sens, j'ai traduit «Dans le creux de sa main»: «en su mano», et «loin des yeux du soleil»: « ocultándola al sol ». Le reste des traducteurs optent pour des solutions similaires, étant donné l'abondance de mots monosyllabiques français dans ces vers.

\section{BIBLIOGRAPHIE}

Éditions et traductions espagnoles des Fleurs du mal:

Les Fleurs du mal, introduction et notes de Vincenette Pichois, Paris, 10/18, 1994.

Las Flores del mal, version espagnole d'Antonio Martínez Sarrión, Madrid, Alianza Editorial, 1977. Las flores del mal, edition bilingüe d'Alain Verjat et Luis Martínez de Merlo, Madrid, Cátedra, 1995. Obras de Charles Baudelaire, étude préliminaire, traduction et notes de Nydia Lamarque, Madrid, Aguilar, 1963.

\section{NOTES}

1. O. Paz, Los hijos del limo, Barcelona, Seix Barral, 1974, p. 95.

2. R. Darío, Cantos de vida y esperanza dans Obras completas II, Madrid, Aguilar, 1967, p. 50. 
INDEX

Mots-clés : Baudelaire (Charles), Fleurs du mal, traduction en espagnol, Correpondances,

Ennemi, Tristesses de la lune 